\title{
Some Features of Flow and Particle Transport in Porous Structures
}

\author{
M.Aydin $^{1,2^{*}}$, G. Balik ${ }^{2}$, A. F. Miguel ${ }^{1}$ and A. H. Reis ${ }^{1}$ \\ ${ }^{1}$ Geophysics Centre of Evora, University of Evora, Rua Romao Ramalho, 59, 7000-671 Evora, Portugal. \\ *e-mail:maydin@uevora.pt \\ ${ }^{2}$ Dept. Mech. Eng., Istanbul Technical University, 34439 Gumussuyu, Istanbul, Turkey
}

\begin{abstract}
There has been a growing interest in the study of porous and complex flow structures due to its impact in technology. This concerns not only environmental but also diagnostic and therapeutic exposure in medical research. Physics of flow within porous structures is especially important to model transport and deposition of viruses, pollutants and drugs deep in these structures. In this work we analyze numerically low and medium Reynolds number flows in axisymmetric cylindrical duct surrounded by a torus. We also consider three different particle sizes $(0.02,0.1$ and $20 \mu \mathrm{m})$ for possible physiological and environmental applications.
\end{abstract}

\section{Introduction}

The study of porous flow structures is of considerable importance in many fields of science and technology [1]. Examples include fields such as energy, biotechnology, environmental sciences etc. The study of these flow structures will help to understand more about fuel cells, particle filters and respiratory tree.

A fuel cell is an electrochemical device that basically consists of two porous electrodes sandwiched around an electrolyte. Oxygen passes over one porous electrode keeping it electronegative while fuel (usually hydrogen) flows over the other (anode). The result of the electrochemical oxidation of fuel is electricity, water and heat. Porous electrodes are crucial to fuel cell performance since they provide high surface area for electrochemical reaction to occur. Microscopic assessment of porous electrodes allows the study in detail these processes in order to improve fuel cell design [2-3].

Porous filters have been used for removing particles with success for many years [4]. Their wide application in air pollution control and in different technologies is due to their reliability in the separation of particles and relatively low operating cost. One of the most important issues in filtration is to know how media's physical properties varied during operating conditions. Filter's collectors (microscopic scale) are properly designed if, during a reasonably long filtering operation, filter's collection efficiency is high and media's flow resistance is below assumed value [5-6]. Description of flow field and particle motion near the filter's collector, as well as, the knowledge of particle cake dynamics provide an important information for a properly design of filter structure.

Inhalation of various particulate pollutants has often been reported to cause short and long term health effects [7-8]. Special attention should be given on the alveolar region where gas-exchange occurs [9]. In order to define strategies for attacking this problem, of particular interest is the study of the patterns of deposition in the alveolar region.

The cases presented motivated this research. There has been many work regarding fluid flow and heat transfer in porous structures in the literature. Most of these studies are viewed as macro structure composed pores. In this work, we take another direction and focus on a pore and its surrounding itself. In this paper we analyze both the velocity and temperature fields, at various Reynolds numbers within and close to a pore considered as an axisymmetric torus. We analyze also particle transportation and deposition on the walls influenced by Reynolds number and particle dimension.

The analysis of the velocity and the temperature fields under imposed heat flux at the surface may help to understand practical cases when a chemical reaction occurs on a catalytic surface (e.g. fuel cells, chemical reactors). On the other hand, the study of particle transport and deposition may illuminate particle deposition in biological flow trees (e.g. bronchial tree). 


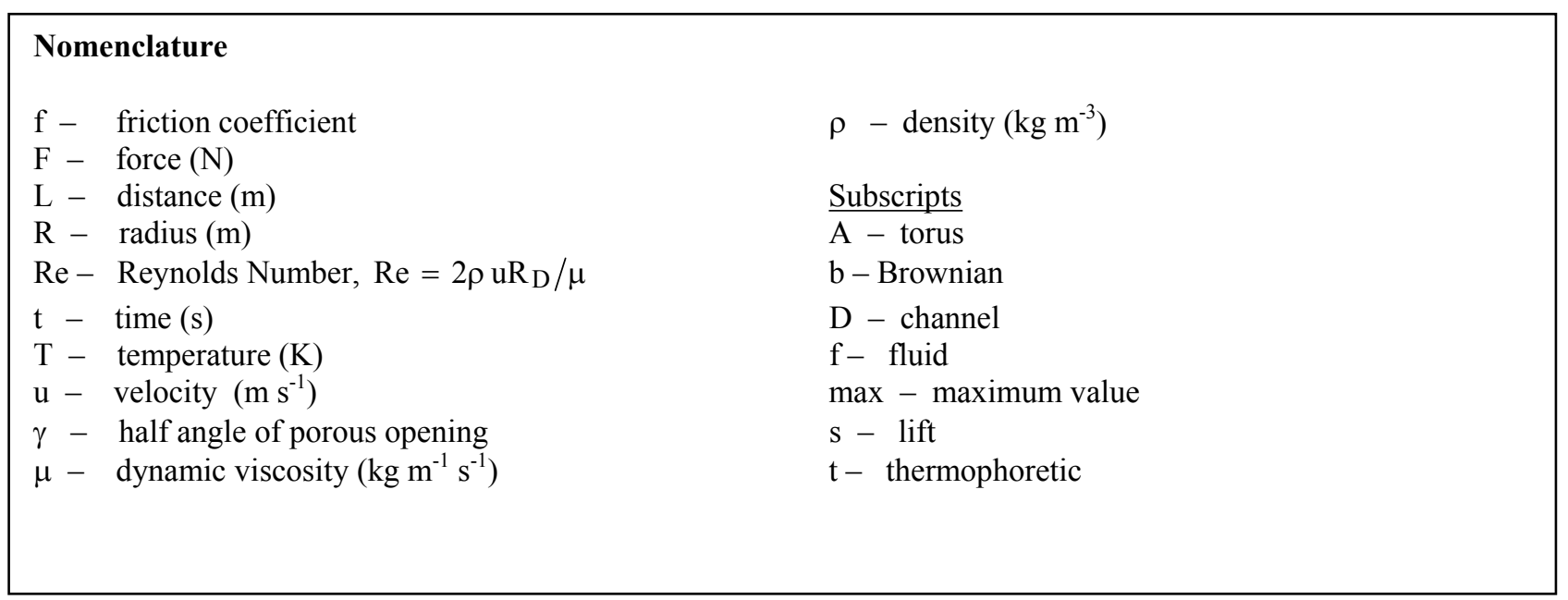

\section{The model}

The geometric model is shown in Fig.1. The geometry used in the flow simulations of [10-11] was adapted here. It consists of a cylindrical duct with radius $\mathrm{R}_{\mathrm{D}}$ surrounded by a torus. The ratio of duct radius to porous radius $\mathrm{R}_{\mathrm{D}} / \mathrm{R}_{\mathrm{A}}=1.25$, the ratio of distance from the central point to inlet to one from exit is $\mathrm{L}_{1} / \mathrm{L}_{2}=1$ and half angle of the porous opening $\gamma=60^{\circ}$. The model is assumed to be three dimensional and axisymmetric.

\section{The Solution}

The steady state flow field is defined by the full Navier-Stokes equations and solved numerically with the finite volume method. The commercial code FLUENT [12] is employed in this study. The non-slip boundary conditions are set along the duct and torus walls. The developed velocity profile is prescribed at the inlet while outflow boundary conditions are set at the exit.

The cell size of the grid where high gradients of velocities are expected has been varied to ensure a grid independent solution.

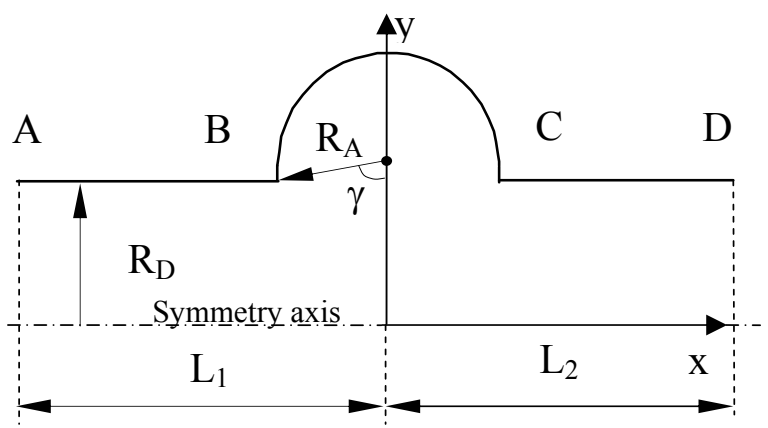

Fig.1 Geometry of the porous structure
The grid with 8184 quadrilateral cells and 8575 nodes are found to be appropriate for present study. Its part around the torus is shown in Fig.2. Reynolds number Re is calculated according to $\operatorname{Re}=2 \rho u R_{D} / \mu$ where $\rho$ is density of air, $u$ is averaged velocity at the inlet, $R_{D}$ is the radius of the channel, and $\mu$ is dynamic viscosity of air.

Solution for low Reynolds number converges rapidly and monotonically. It takes around 400 iterations for $\mathrm{Re}=0.5$. We especially choose $\mathrm{Re}=0.5$ due to availability of a numerical solution in the literature [10]. Variations of residuals for continuity and two components of velocity with iterations are shown in Fig. 3

Streamlines in the pore region are shown in Fig.4. A separation streamline and a slowly re-circulating region within the pore are clearly seen. The flow structures exactly reflects the corresponding flow streamline topology found in the literature (see Fig.5)

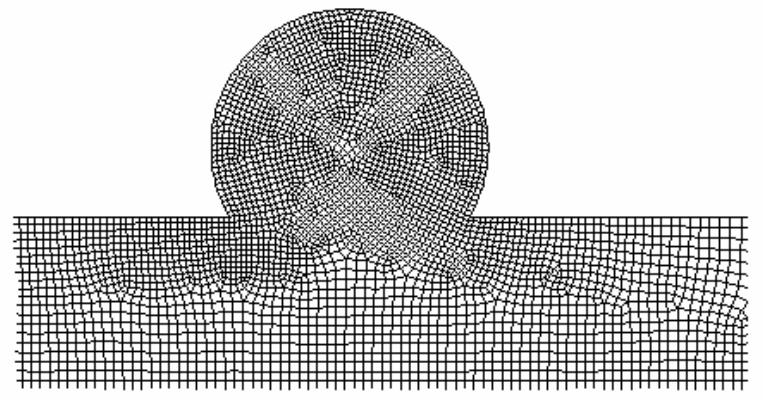

Fig.2 Grid around the pore 


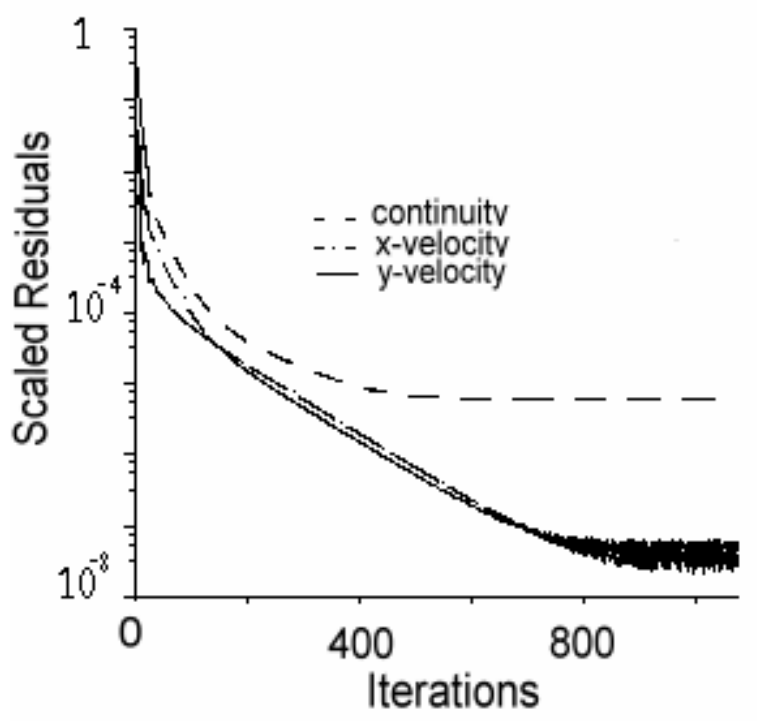

Fig.3 Residuals via iterations

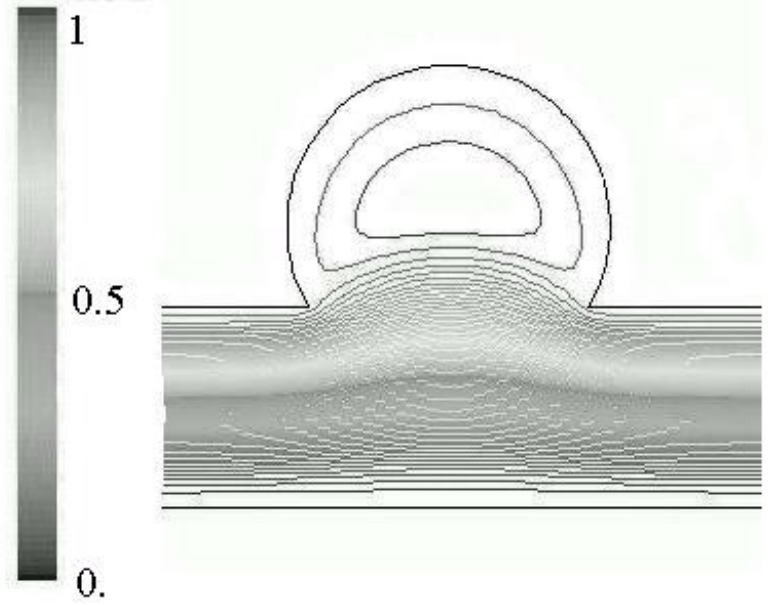

Fig.4 Contours of normalized stream function in the pore at $\mathrm{Re}=0.5$.

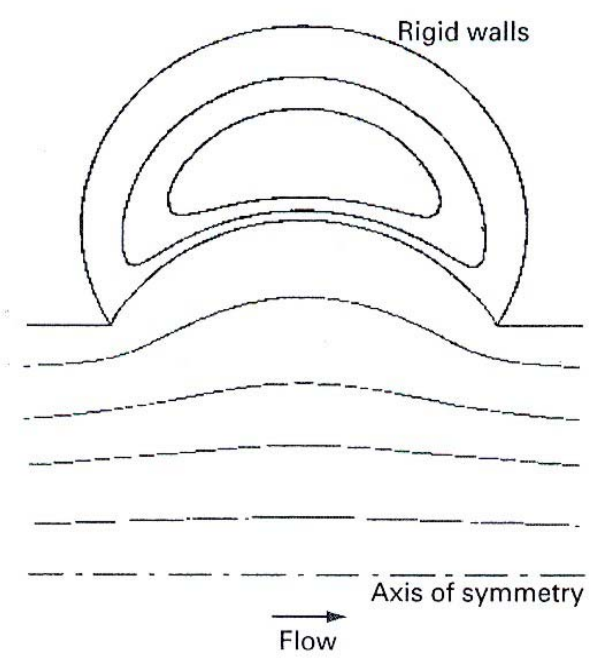

Fig.5 Flow field in the pore by Tippe and Tsuda [10].
We also consider flows at different Re numbers. Velocity vectors at different cross sections of the channel are shown around the pore in Fig 6. Right after the pore, the flow almost fully develops in the case of $\mathrm{Re}=5$. As can be seen from Fig 7, the flow loses its symmetry in the pore region when $\mathrm{Re}$ number increases. The center of vortex moves to right close to the wall. It is also noted that fluid streamlines become more rectilinear with increase in Re number.

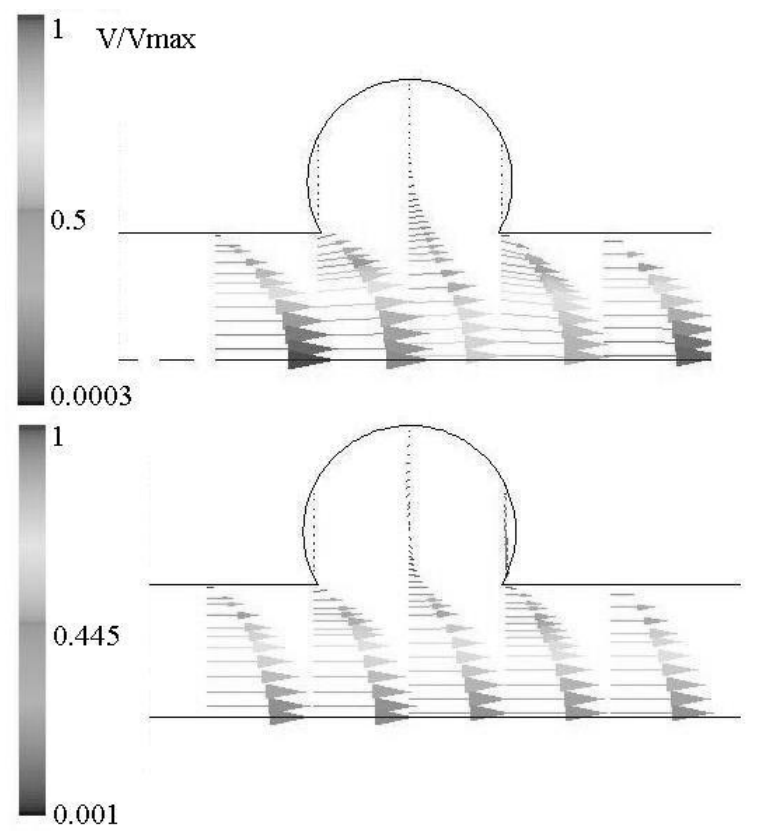

Fig.6 Normalized velocity vectors at different cross sections of the channel: At $\operatorname{Re}=5$ (top) and $\mathrm{Re}=500$ (bottom)

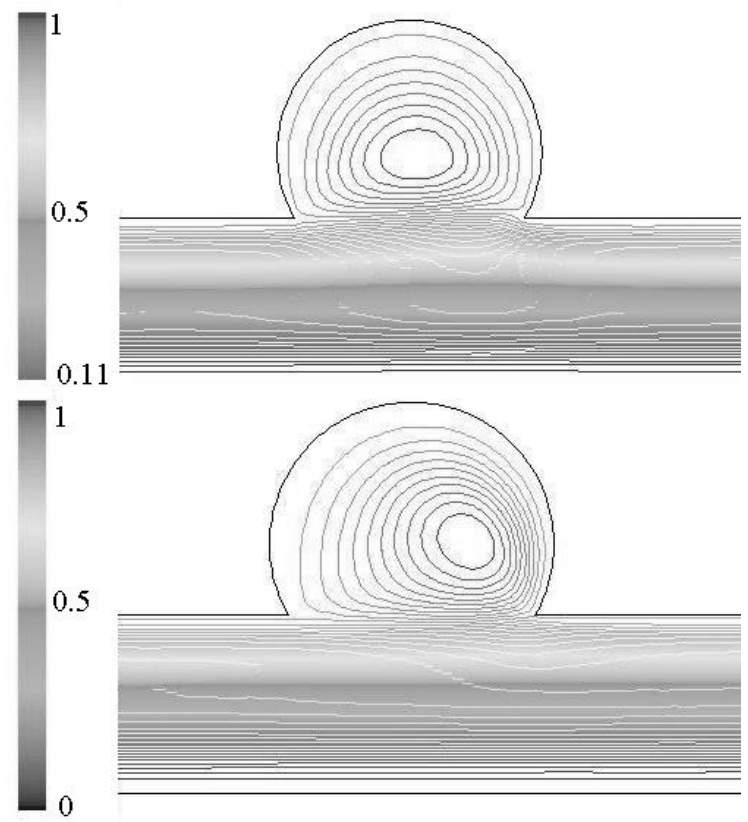

Fig.7 Contours of normalized stream function in the pore: At $\mathrm{Re}=50$ (top) and $\mathrm{Re}=500$ (bottom). 
Energy Equation is also included later to treat forced convection problems. For this purpose, we consider a constant heat flux $\left(0.1 \mathrm{~W} / \mathrm{m}^{2}\right)$ over the surface of the pore while fluid enters the duct at $293 \mathrm{~K}$. Along the surface of the duct constant temperature boundary conditions (293 $\mathrm{K})$ are set. Numerical results in non-dimensionalised form are shown for $\mathrm{Re}=0.5,5$ and 500 in Fig. 8 .

Increase in $\mathrm{Re}$ number shows that isotherms are progressively affected by the flow field. At $\mathrm{Re}=0.5$ they are almost symmetrical with respect to the plane that cuts perpendicularly through the middle of the torus. At $\mathrm{Re}=5$ isotherm asymmetry becomes already noticeable while at $\mathrm{Re}=500$ we see that a significant temperature difference develops between the left and right parts of the torus. We note that this effect happens under constant heat flux over the surface.

We analyze now the case when an exothermic reaction occurs at the surface and we consider both the flow and temperature fields (see Figs. 7 and 8). We see that in the right part of the torus, convection becomes more effective in delivering the reactant to the surface therefore enhancing the heat generation and the heat flux over the surface. It is expected that the temperature field is affected accordingly, i.e. lowering the temperature difference between the left and right parts shall be perceptible.
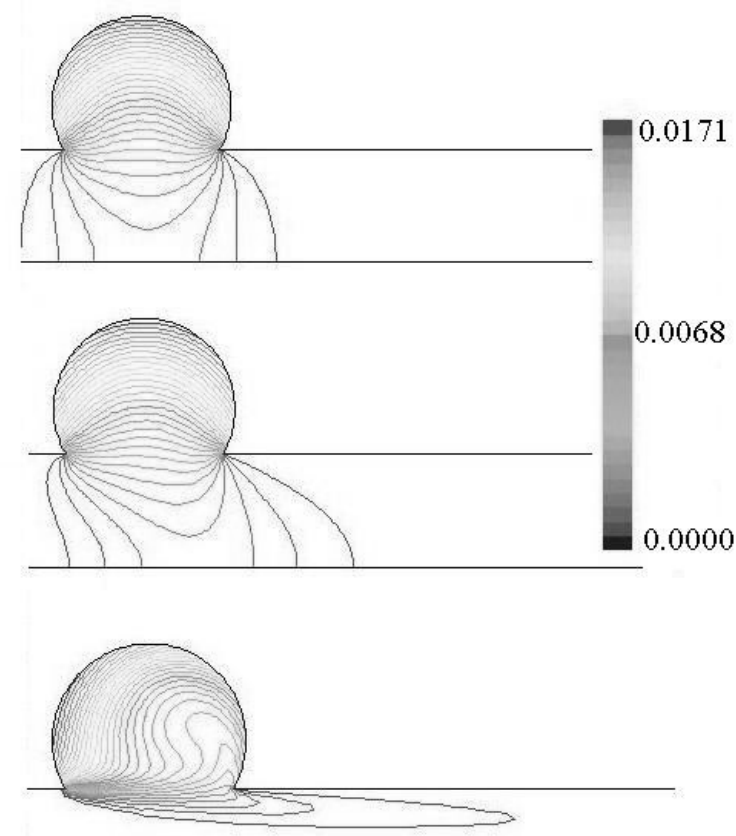

Fig. 8 Non-dimensionalized temperature $\left(T-T_{\mathrm{f}}\right) / \mathrm{T}_{\mathrm{f}}$ contours around the pore: $\mathrm{At} \mathrm{Re}=0.5$ (top), $\mathrm{Re}=5$ (middle) and $\mathrm{Re}=500$ (bottom)

\section{Particle Dynamics}

The motion of a spherical particle of diameter $d$ and mass is described by the equation

$$
\mathrm{m}(\mathrm{du} / \mathrm{dt})=-\mathrm{f}\left(\overrightarrow{\mathrm{u}}-\overrightarrow{\mathrm{u}}_{\mathrm{f}}\right)+\overrightarrow{\mathrm{F}}_{\mathrm{s}}+\overrightarrow{\mathrm{F}}_{\mathrm{b}}(\mathrm{t})+\overrightarrow{\mathrm{F}}_{\mathrm{t}}
$$

This equation represents a force balance on the particle, where mass times acceleration (left-hand side) is the resultant of forces acting on a particle (right-hand side). The first term on the right-hand side represents the viscous drag force due to the motion of the particle relative to the motion of the surrounding fluid. The friction coefficient $\mathrm{f}=3 \pi \mu \mathrm{d} / \mathrm{C}_{\mathrm{S}}$ is assumed to be governed by Stoke's law corrected by the Cunningham factor. The second term is a lift force due to shear. The third term denotes a random force $\mathrm{F}(\mathrm{t})$ arising from Brownian collisions. Finally, the fourth term represents thermophoretic force due to temperature gradient [10, $12]$.

At the mouth of the pore along a vertical surface, particles are injected through the flow. Three different particle sizes $(0.02,0.1$ and $20 \mu \mathrm{m})$ are considered at $\mathrm{Re}=0.5$.

It is observed that fluid velocity near the pore is 10 times lower than one at the centre. Residence time of the particles which follow upper-streams is high and therefore, they are also more likely to get deposited on the walls. Trajectories of the particles are greatly dependent upon initial position.

The number of particles deposited and escaped is documented in Table 1. It is seen that when size of the particles gets bigger, probability to escape is higher for both Re numbers. Smaller particles have a better chance to get deposited in the pore region due to Brownian motion. High Reynolds number flows reduce the probability of deposition for all particles.

At $\mathrm{Re}=0.5$ trajectories of particles of size $20 \mu \mathrm{m}$ are shown in Fig. 9. As the only force that pushes particles towards the axis of the channel is the lift force $F_{s}$ (Eq. 1) we conclude that combination of drag and thermophoretic forces is dominant at low $\mathrm{Re}$ since the Brownian force only induces small perturbation in particles trajectories (see Fig. 10). The situation changes drastically at higher $\mathrm{Re}$ as can be observed in Fig. 11. Here we see that trajectories are curved towards the axis of the channel, therefore proving the dominance of the lift force over other forces.

Table. 1 The number of particle deposited and escaped (\%)

\begin{tabular}{|l|l|l|l|l|l|l|l|l|}
\hline \multirow{2}{*}{$\begin{array}{l}\text { Size } \\
(\mu \mathrm{m})\end{array}$} & \multicolumn{4}{|c|}{$\mathrm{Re}=0.5$} & \multicolumn{4}{c|}{$\mathrm{Re}=500$} \\
\cline { 2 - 9 } & $\mathrm{AB}$ & $\mathrm{BC}$ & $\mathrm{CD}$ & $\mathrm{Esc}$ & $\mathrm{AB}$ & $\mathrm{BC}$ & $\mathrm{CD}$ & $\mathrm{Esc}$ \\
\hline 0.02 & 4 & 8 & 21 & 67 & 0 & 0 & 4 & 96 \\
\hline 0.1 & 0 & 4 & 8 & 88 & 0 & 0 & 4 & 96 \\
\hline 20 & 8 & 0 & 0 & 92 & 0 & 0 & 0 & 100 \\
\hline
\end{tabular}




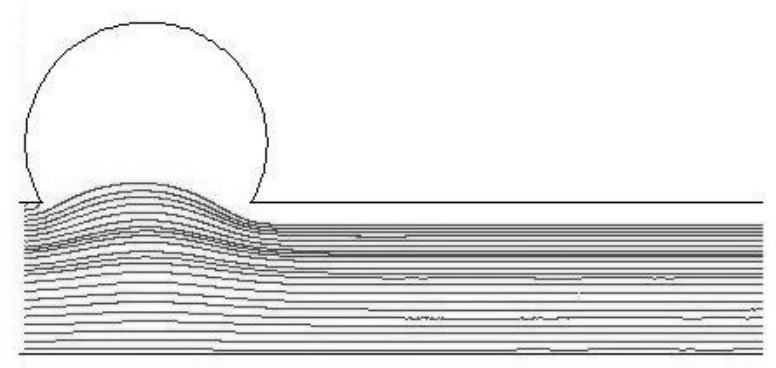

Fig. 9 Particle trajectories of particles of size $20 \mu \mathrm{m}$ at $\mathrm{Re}=0.5$

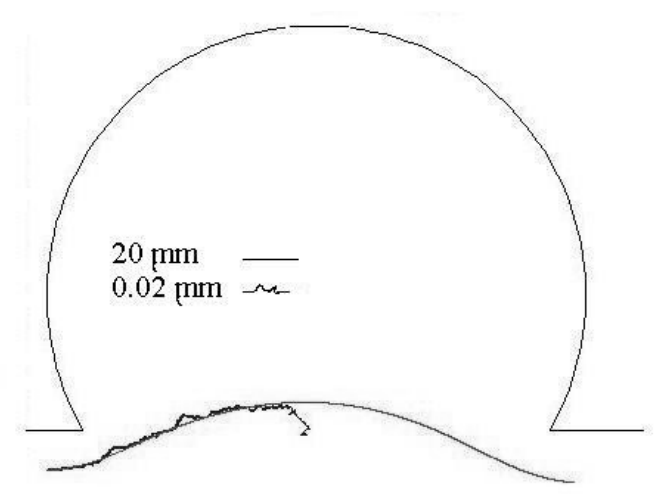

Fig.10 Typical trajectories of 2 particles of size $20 \mu \mathrm{m}$ and $0.02 \mu \mathrm{m}$ at $\mathrm{Re}=0.5$

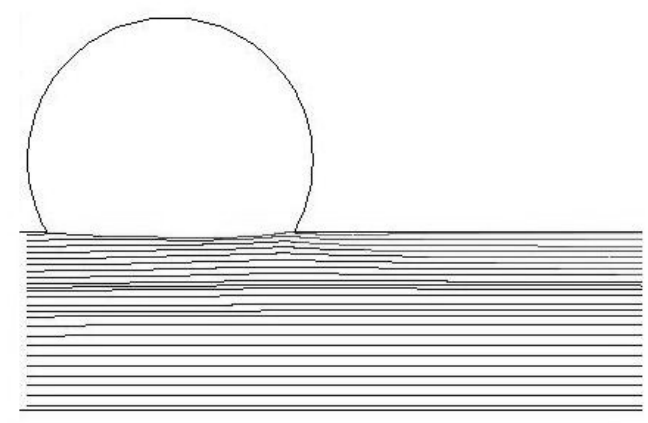

Fig.11 Particle trajectories of particles of size $20 \mu \mathrm{m}$ at $\mathrm{Re}=500$

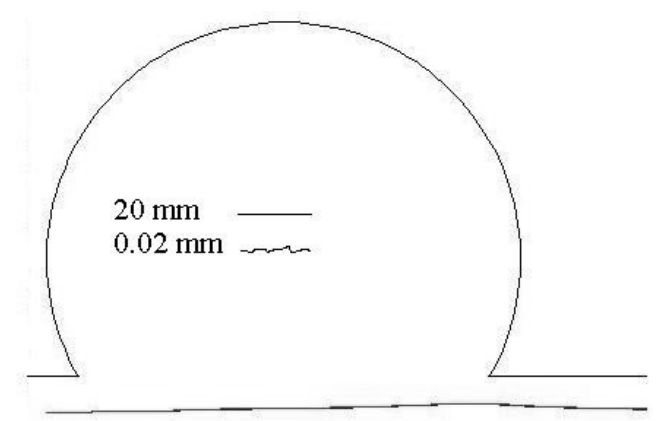

Fig. 12 Typical trajectories of 2 particles of size $20 \mu \mathrm{m}$ and $0.02 \mu \mathrm{m}$ at $\mathrm{Re}=500$
By comparing Figs. (10) and (12) we conclude that the Brownian force becomes negligible at high $\mathrm{Re}$ as compared to others. However, as low Re flows are of characteristic of respiration we see how important the Brownian force can be in particle deposition in the bronchial tree.

\section{Conclusions}

The analysis carried out in this paper has shown how both the velocity and temperature fields in a channel with an axisymmetric pore change with Reynolds number $(\mathrm{Re})$ under prescribed heat flux over the pore surface. At low Re both fields are almost symmetrical with respect to the plane that cuts perpendicularly through the middle of the torus. As Re increases asymmetry develops in both velocity and temperature fields with decrease in temperature in the flow direction and vortex enhancing in the right part of the torus.

When particles are allowed into the flow at low Re $(\mathrm{Re}=0.5)$ we found that particle trajectories are pushed into the pore therefore indicating dominance of drag and thermophorectic forces over lift forces arising from the shear. On the other hand, at $\mathrm{Re}=500$ the lift forces clearly dominate over the other forces this effect being noticeable through the inversion of the curvatures of the trajectories that, in this case, are pushed towards the axis of the channel.

Brownian forces were found to be noticeable only at low $\mathrm{Re}$ flows as is the case of respiration and they may play a significant role in particle deposition in the lungs.

Most of the results presented here may also help in the analysis of flows where a reactant in a porous material is delivered to a catalytic surface where a chemical reaction occurs. This is the case of fuel cells and some chemical reactors.

\section{Acknowledgement}

The authors would like to acknowledge Foundation for the Science and Technology (FCT) for supporting this work (project POCTI/33012/EME/2000).

\section{References}

1. A. Bejan, I. Dincer, S. Lorente, A. F. Miguel and A.H. Reis, Porous and complex flow structures in modern technologies, (2004), Springer-Verlag, New York

2. A. H. Reis, Thermodynamics of fluids in mesoporous media, in: Emerging Technologies and Techniques in Porous Media, D. B. Ingham et al. (eds), 465-431 (2004) Kluwer Academic Publishers

3. A. H. Reis, A. F. Miguel and M. Aydin, Optimized design of a porous structure for gas transport from opening to surface using constructal theory, Proceedings of International Conference on Applications of Porous Media, (2004) ICAPM, Evora Portugal (in press) 
4. A. F. Miguel, Porous Media and Filtration, in: Emerging Technologies and Techniques in Porous Media, D. B. Ingham et al. (eds) 419-431 (2004), Kluwer Academic Publishers.

5. A. F. Miguel, Effect of Air Humidity on the Evolution of Permeability and Performance of a Fibrous Filter during Loading with Hygroscopic and Nonhygroscopic Particles, Journal of Aerosol Science 34, (2003) 783-799.

6. A. F. Miguel, A. H. Reis and M. Aydin, Dynamic loading of porous filters with fine particles, Proceedings of International Conference on Applications of Porous Media, (2004) ICAPM, Evora, Portugal (in press)

7. J. Schwartz and D. W. Dockery, Increased mortality in Philadelphia associated with daily air pollution concentration, Am. Rev. Respir. Dis. 145, 600 (1992).
8. A. F. Miguel, A. H. Reis M. Aydin, A. M. Silva, Particle deposition in airway bifurcations in different breathing conditions, Proceedings of European Aerosol Conference 2004, (in press)

9. A. H. Reis, A. F. Miguel and M. Aydin Constructal theory of flow architecture of the lungs, Medical Physics 31, 1135-1140 (2004)

10. A. Tippe and A. Tsuda, Recirculating flow in an expanding alveolar model: experimental evidence of flow-induced mixing of aerosols in the pulmonary acinus, Journal of Aerosol Science 31, 8 (1999) 979986.

11. A. Tsuda, W. J. Federspiel, P. A. Grant Jr, J. F. Fredberg, Axial dispersion of inert species in alveolated channels, Chemical Engineering Science 46, (5-6) (1991) 1419-1426.

12. FLUENT 6 User's Guide, Fluent Inc. (2003) 